\title{
Le corps des séries de Puiseux généralisées
}

\author{
par \\ Bruno Deschamps (Saint-Etienne)
}

1. Corps des séries de Puiseux. Si $K$ désigne un corps commutatif, le corps $K(X)$ des fractions rationnelles à coefficients dans $K$ est un corps valué, par la valuation usuelle associée aux polynômes (valuation associée au premier $X$ ). On sait qu'alors $K(X)$ n'est pas un corps complet et que son complété est le corps $K((X))$ des séries de Laurent. $K((X))$ est alors muni d'une valuation discrète $\nu$ qui étend la valuation de $K(X)$. On définit $\nu\left(\sum_{k \geq k_{0}} a_{k} X^{k}\right)=k_{0}\left(\right.$ pour $\left.a_{k_{0}} \neq 0\right)$.

Pour tout entier $n \neq 0$, on pose $K_{n}=K\left(\left(X_{n}\right)\right.$ ) (corps de séries de Laurent en la variable $\left.X_{n}\right)$. Pour tout couple d'entiers $(n, m)$ tel que $n$ divise $m$ (disons $m=a n$ ), on définit l'application $\varphi_{n, m}: K_{n} \rightarrow K_{m}$ de la manière suivante : à une série $\sum_{k \geq k_{0}} a_{k} X_{n}^{k}$ on associe la série $\sum_{k \geq k_{0}} a_{k} X_{m}^{a k}$. Les applications $\varphi_{n, m}$ sont clairement des homomorphismes de corps et la donnée des $K_{n}$ et des $\varphi_{n, m}$ forme visiblement un système inductif.

Pour $n=1$, on note plus simplement $K\left(\left(X_{1}\right)\right)=K((X))$. Il apparaît alors que le corps $K_{n}$ est un corps de rupture du polynôme $P(T)=T^{n}-X$ sur $K((X))$, de même si $m=a n$, alors $K_{m}$ est un corps de rupture du polynôme $P(T)=T^{a}-X$ sur $K_{n}$. Ceci justifie alors la notation $X_{n}=X^{1 / n}$ que nous emploierons désormais. Les corps $K_{n}$ sont donc des extensions finies de $K((X))$. On définit alors :

DÉfinition. On appelle corps des séries de Puiseux à coefficients dans $K$ le corps noté Puis $(K)$ obtenu en prenant la limite inductive du système de corps et d'homomorphismes $\left(K_{n}, \varphi_{n, m}\right)$.

Ainsi, le corps Puis $(K)$ est une extension algébrique de $K((X))$. Par ailleurs, Puis $(K)$ possède une unique valuation qui prolonge celle de $K((X))$ (puisque $K((X))$ est complet à valuation discrète). Nous continuerons à noter cette valuation $\nu$. Si $\sum_{k \geq k_{0}} a_{k} X^{k / n}$ désigne une série de Puiseux $\left(a_{k_{0}} \neq 0\right)$, on a alors $\nu\left(\sum_{k \geq k_{0}} a_{k} X^{k / n}\right)=k_{0} / n$. Le corps résiduel de Puis $(K)$

2000 Mathematics Subject Classification: Primary 12E30; Secondary 12F20. 
est alors $K$. Notons que $\nu$ n'est plus discrète. Dans cet article, pour tout corps $k$, on notera $\bar{k}$ la clôture algébrique $k$. L'intérêt premier de l'étude de $\operatorname{Puis}(K)$ vient du théorème suivant (voir $[$ Ser $]$ ) :

ThÉORÈme. Si K est un corps algébriquement clos de caractéristique nulle, alors

$$
\overline{K((X))}=\operatorname{Puis}(K) \text {. }
$$

Une ombre persiste tout de même au tableau :

Proposition 1. Puis $(K)$ n'est jamais un corps complet pour $\nu$.

Pr e u ve. En effet, la suite $S_{n}(X)=\sum_{k=1}^{n} X^{k+1 / k}$ de séries de Puiseux est visiblement de Cauchy. Supposons que $\left(S_{n}\right)_{n}$ converge dans Puis $(K)$, disons vers $S=\sum_{k \geq k_{0}} a_{k} X^{k / n_{0}}$. Considérons un nombre premier $p$ ne divisant pas $n_{0}$; alors $\nu\left(S-S_{n}\right) \leq\left(p^{2}+1\right) / p$ pour tout $n \geq p$, ce qui rend impossible le fait que $\lim _{n} \nu\left(S-S_{n}\right)=+\infty$.

On veut donc compléter Puis $(K)$. Pour cela, on va généraliser le corps des séries de Puiseux.

\section{Corps des séries de Puiseux généralisées}

2.1. Construction. On considère l'ensemble

$$
\mathcal{A}=\left\{\left(\alpha_{n}\right)_{n} \in \mathbb{Q}^{\mathbb{N}}: \forall n \in \mathbb{N}, \alpha_{n+1}>\alpha_{n} \text { et } \lim _{n \rightarrow+\infty} \alpha_{n}=+\infty\right\}
$$

des suites de rationnels strictement croissantes et tendant vers $+\infty$.

On considère aussi les deux ensembles suivants :

$$
\mathcal{O}=\{P \subset \mathbb{Q}: P \text { sans point d'accumulation, } \inf (P) \in \mathbb{R}, \sup (P)=+\infty\}
$$

et

$$
\mathcal{O}^{\prime}=\{P \subset \mathbb{Q}: \sharp P=+\infty \text { et } \forall A \in \mathbb{R}, \sharp(]-\infty, A[\cap P)<+\infty\} .
$$

Soit alors $\Delta: \mathcal{A} \rightarrow \mathcal{P}(\mathbb{R})$ définie par

$$
\forall\left(\alpha_{n}\right)_{n} \in \mathcal{A}, \quad \Delta\left(\left(\alpha_{n}\right)_{n}\right)=\left\{\alpha_{n}: n \in \mathbb{N}\right\} .
$$

Les deux lemmes qui suivent sont des conséquences immédiates du théorème de Bolzano-Weierstrass :

Lemme 1. $\mathcal{O}=\mathcal{O}^{\prime}$ et $\Delta$ est une bijection de $\mathcal{A} \operatorname{sur} \mathcal{O}$.

Lemme 2. L'ensemble $\mathcal{O}$ est stable par réunions finies.

De même, pour la somme, on a :

Lemme 3. Soit $P_{1}, P_{2} \in \mathcal{O}$, alors

$$
P_{1}+P_{2}=\left\{x+y: x \in P_{1}, y \in P_{2}\right\} \in \mathcal{O} .
$$

Sur $\mathcal{A}$, on définit une loi de composition interne + de la manière suivante :

$$
\left(\alpha_{n}\right)_{n}+\left(\beta_{n}\right)_{n}=\Delta^{-1}\left(\Delta\left(\left(\alpha_{n}\right)_{n}\right)+\Delta\left(\left(\beta_{n}\right)_{n}\right)\right) .
$$


De plus, on définit une relation binaire $\leq$ de la manière suivante :

$$
\forall\left(\alpha_{n}\right)_{n},\left(\beta_{n}\right)_{n} \in \mathcal{A}, \quad\left(\alpha_{n}\right)_{n} \leq\left(\beta_{n}\right)_{n} \Leftrightarrow \Delta\left(\left(\alpha_{n}\right)_{n}\right) \subset \Delta\left(\left(\beta_{n}\right)_{n}\right) .
$$

Lemme 4. L'ensemble $(A, \leq)$ est un ensemble ordonné, réticulé supérieurement (donc en particulier filtrant à droite).

Preuve. La relation $\leq$ est clairement réflexive et transitive, le fait qu'elle soit antisymétrique provient de l'injectivité de $\Delta$.

Soient $\left(\alpha_{n}\right)_{n}$ et $\left(\beta_{n}\right)_{n}$ deux éléments de $\mathcal{A}$; alors la suite

$$
\left(\gamma_{n}\right)_{n}=\Delta^{-1}\left(\Delta\left(\left(\alpha_{n}\right)_{n}\right) \cup \Delta\left(\left(\beta_{n}\right)_{n}\right)\right)
$$

est clairement la borne supérieure dans $\mathcal{A}$ de la partie $\left\{\left(\alpha_{n}\right)_{n},\left(\beta_{n}\right)_{n}\right\}$.

Pour une suite $\left(\alpha_{n}\right)_{n} \in \mathcal{A}$, on pose

$$
\mathcal{A}_{\left(\alpha_{n}\right)_{n}}=\left\{\left(u_{\alpha_{n}}\right)_{n} \in K^{\mathbb{N}}\right\} .
$$

C'est l'ensemble des suites d'éléments de $K$ indicées par la suite $\left(\alpha_{n}\right)_{n}$. Dans la suite on notera un élément $\left(u_{\alpha_{n}}\right)_{n} \in \mathcal{A}_{\left(\alpha_{n}\right)_{n}}$ de la manière suivante :

$$
\sum_{n \geq 0} u_{\alpha_{n}} X^{\alpha_{n}}
$$

Soient $\left(\alpha_{n}\right)_{n}$ et $\left(\beta_{n}\right)_{n}$ deux éléments de $\mathcal{A}$ tels que $\left(\alpha_{n}\right)_{n} \leq\left(\beta_{n}\right)_{n}$. On définit une application $\Phi_{\left(\alpha_{n}\right)_{n},\left(\beta_{n}\right)_{n}}: \mathcal{A}_{\left(\alpha_{n}\right)_{n}} \rightarrow \mathcal{A}_{\left(\beta_{n}\right)_{n}}$ en posant

$$
\Phi_{\left(\alpha_{n}\right)_{n},\left(\beta_{n}\right)_{n}}\left(\sum_{n \geq 0} u_{\alpha_{n}} X^{\alpha_{n}}\right)=\sum_{m \geq 0} u_{\beta_{m}} X^{\beta_{m}}
$$

avec

$$
u_{\beta_{m}}= \begin{cases}0 & \text { si } \forall n \in \mathbb{N}, \alpha_{n} \neq \beta_{m}, \\ u_{\alpha_{n}} & \text { si } \alpha_{n}=\beta_{m} .\end{cases}
$$

(Cette application est bien définie car si pour un indice $m \in \mathbb{N}$ donné, il existe un indice $n \in \mathbb{N}$ tel que $\alpha_{n}=\beta_{m}$ alors, à cause de la stricte croissance de $\left(\alpha_{n}\right)_{n}$, cet indice est unique pour cette propriété.)

LEMme 5. Le système d'ensembles et d'applications

$$
\left\{\mathcal{A}_{\left(\alpha_{n}\right)_{n}}, \Phi_{\left(\alpha_{n}\right)_{n},\left(\beta_{n}\right)_{n}}\right\}_{\mathcal{A}}
$$

est un système inductif.

Preuve. On a établi que $(\mathcal{A}, \leq)$ était un ensemble filtrant à droite. Il est clair que pour tout $\left(\alpha_{n}\right)_{n} \in \mathcal{A}, \Phi_{\left(\alpha_{n}\right)_{n},\left(\alpha_{n}\right)_{n}}=\operatorname{id}_{\mathcal{A}_{\left(\alpha_{n}\right)_{n}}}$.

Soit $\left(\alpha_{n}\right)_{n},\left(\beta_{n}\right)_{n}$ et $\left(\gamma_{n}\right)_{n}$ trois éléments de $\mathcal{A}$ tels que $\left(\alpha_{n}\right)_{n} \leq\left(\beta_{n}\right)_{n} \leq$ $\left(\gamma_{n}\right)_{n}$. Posons

$$
S=\sum_{n \geq 0} u_{\alpha_{n}} X^{\alpha_{n}}
$$




$$
\begin{aligned}
& \Phi_{\left(\alpha_{n}\right)_{n},\left(\beta_{n}\right)_{n}}(S)=\sum_{m \geq 0} v_{\beta_{m}} X^{\beta_{m}}=S^{\prime}, \\
& \Phi_{\left(\beta_{n}\right)_{n},\left(\gamma_{n}\right)_{n}}\left(S^{\prime}\right)=\sum_{k \geq 0} w_{\gamma_{k}} X^{\gamma_{k}}, \\
& \Phi_{\left(\alpha_{n}\right)_{n},\left(\gamma_{n}\right)_{n}}(S)=\sum_{k \geq 0} w_{\gamma_{k}}^{\prime} X^{\gamma_{k}} .
\end{aligned}
$$

Soit $k \in \mathbb{N}$; deux situations se présentent alors :

1) $\forall n \in \mathbb{N}, \alpha_{n} \neq \gamma_{k}$. Dans ces conditions, $w_{\gamma_{n}}^{\prime}=0$.

1.1) $\forall m \in \mathbb{N}, \beta_{m} \neq \gamma_{k}$. Alors $w_{\gamma_{k}}=0$.

1.2) $\beta_{m_{0}}=\gamma_{k}$. Dans cette situation on a nécessairement $\alpha_{n} \neq \beta_{m_{0}}$ pour tout $n \in \mathbb{N}$, donc $w_{\gamma_{k}}=v_{\beta_{m_{0}}}=0$.

2) $\alpha_{n_{0}}=\gamma_{k}$. Dans ces conditions $w_{\gamma_{k}}^{\prime}=u_{\alpha_{n_{0}}}$. Mais alors il existe $m_{0} \in \mathbb{N}$ tel que $\beta_{m_{0}}=\alpha_{n_{0}}$ et donc $v_{\beta_{m_{0}}}=u_{\alpha_{n_{0}}}$ et comme $\beta_{m_{0}}=\gamma_{k}$, on a $w_{\gamma_{k}}=$ $u_{\alpha_{n_{0}}}=w_{\gamma_{k}}^{\prime}$.

Ainsi, dans tous les cas, on a $w_{\gamma_{k}}=w_{\gamma_{k}}^{\prime}$, donc

$$
\Phi_{\left(\beta_{n}\right)_{n},\left(\gamma_{n}\right)_{n}} \circ \Phi_{\left(\alpha_{n}\right)_{n},\left(\beta_{n}\right)_{n}}=\Phi_{\left(\alpha_{n}\right)_{n},\left(\gamma_{n}\right)_{n}} .
$$

Le système est bien inductif.

On pose alors $\Omega=\lim _{\longrightarrow}\left(\mathcal{A}_{\left(\alpha_{n}\right)_{n}}, \Phi_{\left.\left(\alpha_{n}\right)_{n},\left(\beta_{n}\right)_{n}\right) \mathcal{A}}\right.$. Rappelons que $\Omega$ est l'ensemble quotient $\amalg \overrightarrow{\mathcal{A}_{\left(\alpha_{n}\right)_{n}}} / \mathcal{R}$ où $\mathcal{R}$ est la relation d'équivalence suivante : $S \mathcal{R} S^{\prime}\left(\right.$ avec $S \in \mathcal{A}_{\left(\alpha_{n}\right)_{n}}$ et $\left.S^{\prime} \in \mathcal{A}_{\left(\beta_{n}\right)_{n}}\right)$ si et seulement s'il existe $\left(\gamma_{n}\right)_{n} \in \mathcal{A}$ majorant $\left(\alpha_{n}\right)_{n}$ et $\left(\beta_{n}\right)_{n}$ tel que

$$
\Phi_{\left(\alpha_{n}\right)_{n},\left(\gamma_{n}\right)_{n}}(S)=\Phi_{\left(\beta_{n}\right)_{n},\left(\gamma_{n}\right)_{n}}\left(S^{\prime}\right) .
$$

Lemme 6. Soit $S=\sum_{n \geq 0} u_{\alpha_{n}} X^{\alpha_{n}}$ et $S^{\prime}=\sum_{n \geq 0} v_{\beta_{n}} X^{\beta_{n}}$ deux éléments de $\mathcal{A}_{\left(\alpha_{n}\right)_{n}}$. Les propositions suivantes sont équivalentes :

(i) $S \mathcal{R} S^{\prime}$.

(ii) $\forall r \in \Delta\left(\left(\alpha_{n}\right)_{n}\right) \cap \Delta\left(\left(\beta_{n}\right)_{n}\right), u_{r}=v_{r} ; \forall r \in \Delta\left(\left(\alpha_{n}\right)_{n}\right)-\Delta\left(\left(\beta_{n}\right)_{n}\right)$, $u_{r}=0$ et $\forall r \in \Delta\left(\left(\beta_{n}\right)_{n}\right)-\Delta\left(\left(\alpha_{n}\right)_{n}\right), v_{r}=0$.

Preuve. (i) $\Rightarrow\left(\right.$ ii) : Soit $\left(\gamma_{n}\right)_{n} \in \mathcal{A}$ une suite telle que $\Phi_{\left(\alpha_{n}\right)_{n},\left(\gamma_{n}\right)_{n}}(S)=$ $\Phi_{\left(\beta_{n}\right)_{n},\left(\gamma_{n}\right)_{n}}\left(S^{\prime}\right)$. Posons

$$
\Phi_{\left(\alpha_{n}\right)_{n},\left(\gamma_{n}\right)_{n}}(S)=\sum_{n \geq 0} w_{\gamma_{n}} X^{\gamma_{n}}=\Phi_{\left(\beta_{n}\right)_{n},\left(\gamma_{n}\right)_{n}}\left(S^{\prime}\right) .
$$

Si $r \in \Delta\left(\left(\alpha_{n}\right)_{n}\right) \cap \Delta\left(\left(\beta_{n}\right)_{n}\right)$, alors $u_{r}=w_{r}=v_{r}$. Si maintenant $r \in$ $\Delta\left(\left(\alpha_{n}\right)_{n}\right)-\Delta\left(\left(\beta_{n}\right)_{n}\right)$, alors $u_{r}=w_{r}=0$ car il n'existe pas d'indice $n \in \mathbb{N}$ tel que $\beta_{n}=r$.

(ii) $\Rightarrow$ (i) : Si (ii) est satisfait, il est alors clair que

$$
\Phi_{\left(\alpha_{n}\right)_{n},\left(\gamma_{n}\right)_{n}}(S)=\Phi_{\left(\beta_{n}\right)_{n},\left(\gamma_{n}\right)_{n}}\left(S^{\prime}\right)
$$

$\operatorname{pour}\left(\gamma_{n}\right)_{n}=\sup \left(\left(\alpha_{n}\right)_{n},\left(\beta_{n}\right)_{n}\right)$. 
REMARQUE. On a alors les propriétés suivantes :

- Un élément de $\Omega$ a au maximum un représentant dans chaque $\mathcal{A}_{\left(\alpha_{n}\right)_{n}}$.

- Si $x \in \Omega$ a un représentant dans $\mathcal{A}_{\left(\alpha_{n}\right)_{n}}$, alors $x$ a un représentant dans $\mathcal{A}_{\left(\beta_{n}\right)_{n}}$ pour tout $\left(\beta_{n}\right)_{n} \geq\left(\alpha_{n}\right)_{n}$.

- Si un élément $x$ de $\Omega$ possède un représentant $\sum_{n \geq 0} u_{\alpha_{n}} X^{\alpha_{n}}$ tel que la suite $\left(u_{\alpha_{n}}\right)_{n}$ ne soit pas nulle à partir d'un certain rang, alors $x$ possède un représentant (unique) $\sum_{n>0} u_{\beta_{n}} X^{\beta_{n}}$ tel que pour tout $n \in \mathbb{N}, u_{\beta_{n}} \neq 0$ (on a alors $\left(\beta_{n}\right)_{n} \leq\left(\alpha_{n}\right)_{n}$ ) ce représentant s'appelle alors le "représentant canonique de $x "$.

- Si un élément $x \in \Omega$ possède un représentant canonique dans $\mathcal{A}_{\left(\gamma_{n}\right)_{n}}$, alors la suite $\left(\gamma_{n}\right)_{n}$ est un plus petit élément dans l'ensemble des suites $\left(\alpha_{n}\right)_{n} \in \mathcal{A}$ telles que $x$ admette un représentant dans $\mathcal{A}_{\left(\alpha_{n}\right)_{n}}$.

Dans la suite, si $S \in \amalg \mathcal{A}_{\left(\alpha_{n}\right)_{n}}$, on notera $\bar{S}$ la classe de $S$ dans $\Omega$.

- Sur $\amalg \mathcal{A}_{\left(\alpha_{n}\right)_{n}}$, on définit une loi de composition interne + de la manière suivante : si $S \in \mathcal{A}_{\left(\alpha_{n}\right)_{n}}$ et $S^{\prime} \in \mathcal{A}_{\left(\beta_{n}\right)_{n}}$, alors en posant

$$
\begin{gathered}
\left(\gamma_{n}\right)_{n}=\sup \left(\left(\alpha_{n}\right)_{n},\left(\beta_{n}\right)_{n}\right), \\
\Phi_{\left(\alpha_{n}\right)_{n},\left(\gamma_{n}\right)_{n}}(S)=\sum_{n \geq 0} u_{\gamma_{n}} X^{\gamma_{n}}, \quad \Phi_{\left(\beta_{n}\right)_{n},\left(\gamma_{n}\right)_{n}}\left(S^{\prime}\right)=\sum_{n \geq 0} v_{\gamma_{n}} X^{\gamma_{n}},
\end{gathered}
$$

on définit

$$
S+S^{\prime}=\sum_{n \geq 0}\left(u_{\gamma_{n}}+v_{\gamma_{n}}\right) X^{\gamma_{n}} \in \mathcal{A}_{\left(\gamma_{n}\right)_{n}} .
$$

- Sur $\amalg \mathcal{A}_{\left(\alpha_{n}\right)_{n}}$, on définit aussi une autre loi de composition interne : si $S=\sum_{n \geq 0} u_{\alpha_{n}} X^{\alpha_{n}} \in \mathcal{A}_{\left(\alpha_{n}\right)_{n}}$ et $S^{\prime}=\sum_{n \geq 0} v_{\beta_{n}} X^{\beta_{n}} \in \mathcal{A}_{\left(\beta_{n}\right)_{n}}$, alors en posant $\left(\gamma_{n}\right)_{n}=\left(\alpha_{n}\right)_{n}+\left(\beta_{n}\right)_{n}$, on définit

$$
S \cdot S^{\prime}=\sum_{n \geq 0} W_{\gamma_{n}} X^{\gamma_{n}} \in \mathcal{A}_{\left(\gamma_{n}\right)_{n}}
$$

avec

$$
W_{\gamma_{n}}=\sum_{\alpha_{i}+\beta_{j}=\gamma_{n}} u_{\alpha_{i}} v_{\beta_{j}}
$$

(cette dernière somme étant visiblement finie).

Lemme 7. Si $S, S^{\prime}, T, T^{\prime} \in \amalg \mathcal{A}_{\left(\alpha_{n}\right)_{n}}$ sont tels que $\bar{S}=\overline{S^{\prime}}$ et $\bar{T}=\overline{T^{\prime}}$, alors

$$
\overline{S+T}=\overline{S^{\prime}+T^{\prime}} \text { et } \overline{S \cdot T}=\overline{S^{\prime} \cdot T^{\prime}} .
$$

Preuve. A cause de l'évidente commutativité des lois + et $\cdot$, la proposition énoncée dans ce lemme équivaut à $\overline{S+T}=\overline{S^{\prime}+T}$ et $\overline{S \cdot T}=\overline{S^{\prime} \cdot T}$.

Posons $S+T=\sum_{n>0}\left(u_{\gamma_{n}}+v_{\gamma_{n}}\right) X^{\gamma_{n}} \in \mathcal{A}_{\left(\gamma_{n}\right)_{n}}$ et $S^{\prime}+T=\sum_{n>0}\left(u_{\delta_{n}}^{\prime}+\right.$ $\left.v_{\delta_{n}}^{\prime}\right) X^{\delta_{n}} \in \mathcal{A}_{\left(\delta_{n}\right)_{n}}$. On sait que les séries $\sum_{n \geq 0} u_{\gamma_{n}} X^{\gamma_{n}}$ et $\sum_{n \geq 0} u_{\delta_{n}}^{\prime} X^{\delta_{n}}$ sont en relation ainsi que les séries $\sum_{n \geq 0} v_{\gamma_{n}} X^{\gamma_{n}}$ et $\sum_{n \geq 0} v_{\delta_{n}}^{\prime} X^{\delta_{n}}$. Considérons 
deux indices $n_{0}$ et $n_{1}$ tels que $\gamma_{n_{0}}=\delta_{n_{1}}$; alors $u_{\gamma_{n_{0}}}=u_{\delta_{n_{1}}}^{\prime}$ et $v_{\gamma_{n_{0}}}=v_{\delta_{n_{1}}}^{\prime}$, donc $u_{\gamma_{n_{0}}}+v_{\gamma_{n_{0}}}=u_{\delta_{n_{1}}}^{\prime}+v_{\delta_{n_{1}}}^{\prime}$.

Soit maintenant $n_{0} \in \mathbb{N}$ tel que $\gamma_{n_{0}} \neq \delta_{n}$ pour tout $n \in \mathbb{N}$; alors $u_{\gamma_{n_{0}}}=0$ et $v_{\gamma_{n_{0}}}=0$, c'est-à-dire $u_{\gamma_{n_{0}}}+v_{\gamma_{n_{0}}}=0$. Les séries $S+T$ et $S^{\prime}+T$ sont bien en relation.

Soient $S=\sum_{n \geq 0} u_{\alpha_{n}} X^{\alpha_{n}}, S^{\prime}=\sum_{n \geq 0} u_{\alpha_{n}^{\prime}}^{\prime} X^{\alpha_{n}^{\prime}}$ et $T=\sum_{n \geq 0} v_{\beta_{n}} X^{\beta_{n}}$. Posons $S \cdot T=\sum_{n \geq 0} w_{\gamma_{n}} X^{\gamma_{n}}$ avec $w_{\gamma_{n}}=\sum_{\alpha_{i}+\beta_{j}=\gamma_{n}} u_{\alpha_{i}} v_{\alpha_{j}}$ et $S^{\prime} \cdot T=$ $\sum_{n \geq 0} w_{\gamma_{n}^{\prime}}^{\prime} X^{\gamma_{n}^{\prime}}$ avec $w_{\gamma_{n}^{\prime}}^{\prime}=\sum_{\alpha_{i}^{\prime}+\beta_{j}=\gamma_{n}^{\prime}} u_{\alpha_{i}^{\prime}}^{\prime} v_{\alpha_{j}}$. Soit un indice $n_{0} \in \mathbb{N}$ tel que $\gamma_{n}^{\prime} \neq \gamma_{n_{0}}$ pour tout $n \in \mathbb{N}$. Prenons des indices $i_{0} \in \mathbb{N}$ et $j_{0} \in \mathbb{N}$ tels que $\alpha_{i_{0}}+\beta_{j_{0}}=\gamma_{n_{0}}$. Alors pour tout $i \in \mathbb{N}, \alpha_{i}^{\prime} \neq \alpha_{i_{0}}$ car sinon il existerait $n \in \mathbb{N}$ tel que $\gamma_{n}^{\prime}=\alpha_{i}^{\prime}+\beta_{j_{0}}=\alpha_{i_{0}}+\beta_{j_{0}}=\gamma_{n}$. Ainsi, $u_{\alpha_{i_{0}}}=0$ et par suite $w_{\gamma_{n_{0}}}=\sum_{\alpha_{i}+\beta_{j}=\gamma_{n_{0}}} u_{\alpha_{i}} v_{\alpha_{j}}=0$.

Soient maintenant deux indices $n_{0}$ et $n_{1}$ tels que $\gamma_{n_{0}}=\gamma_{n_{1}}^{\prime}=\omega$. Posons $R=\Delta\left(\left(\alpha_{n}\right)_{n}\right) \cap \Delta\left(\left(\alpha_{n}^{\prime}\right)_{n}\right)$. On a alors

$$
\begin{aligned}
w_{\gamma_{n_{0}}} & =\sum_{\alpha_{i}+\beta_{j}=\gamma_{n_{0}}} u_{\alpha_{i}} v_{\alpha_{j}}=\sum_{r+\beta_{j}=\omega, r \in R} u_{r} v_{\alpha_{j}} \\
& =\sum_{r+\beta_{j}=\omega, r \in R} u_{r}^{\prime} v_{\alpha_{j}}=\sum_{\alpha_{i}^{\prime}+\beta_{j}=\omega} u_{\alpha_{i}^{\prime}}^{\prime} v_{\alpha_{j}} \\
& =\sum_{\alpha_{i}^{\prime}+\beta_{j}=\gamma_{n_{1}}^{\prime}} u_{\alpha_{i}^{\prime}}^{\prime} v_{\alpha_{j}}=w_{\gamma_{n}^{\prime}}^{\prime} .
\end{aligned}
$$

Les séries $S \cdot T$ et $S^{\prime} \cdot T$ sont bien équivalentes.

On vient donc d'établir que les lois + et - sont compatibles avec $\mathcal{R}$. Elles définissent donc des lois de compositions internes sur $\Omega$. On a alors :

Proposition-DÉfinition. L'ensemble $(\Omega,+, \cdot)$ est un corps commutatif que l'on appelle corps des séries de Puiseux généralisées. On le note $\widetilde{\operatorname{Puis}}(K)$.

Preuve. - $(\Omega,+)$ est un groupe abélien. Ce fait vient immédiatement de la structure de groupe abélien de $(K,+)$, le neutre étant par exemple représenté par la série $\sum_{n \geq 0} u_{n} X^{n}$ où $u_{n}=0$ pour tout $n \in \mathbb{N}$.

- La loi - est associative. Soient $R, S, T$ trois éléments de $\Omega$. On choisit un $\left(\alpha_{n}\right)_{n} \in \mathcal{A}$ tel que $R=\sum_{n \geq 0} r_{\alpha_{n}} X^{\alpha_{n}}, S=\sum_{n \geq 0} s_{\alpha_{n}} X^{\alpha_{n}}, T=$ $\sum_{n \geq 0} t_{\alpha_{n}} X^{\alpha_{n}}$. Soit $\left(\gamma_{n}\right)_{n}=\left(\alpha_{n}\right)_{n}+\left(\alpha_{n}\right)_{n}+\left(\alpha_{n}\right)_{n}$. On a alors (après calcul)

$$
(R \cdot S) \cdot T=R \cdot(S \cdot T)=\sum_{n \geq 0}\left(\sum_{\alpha_{p}+\alpha_{q}+\alpha_{h}=\gamma_{n}} r_{\alpha_{p}} s_{\alpha_{q}} t_{\alpha_{h}}\right) X^{\gamma_{n}} .
$$

- La loi est commutative. C'est évident.

- La série $\sum_{n \geq 0} \alpha_{n} X^{n}$ avec $\alpha_{0}=1$ et $\alpha_{n}=0$ pour $n \geq 1$ représente visiblement un neutre de $(\Omega, \cdot)$. 
- Inversibilité. Soit $S \in \Omega$ non nul; on écrit $S=X^{r} \cdot T$ avec $T=$ $\sum_{n>0} t_{\gamma_{n}} X^{\gamma_{n}}$ et $r \in \mathbb{Q}, \gamma_{0}=0$ et $t_{\gamma_{0}} \neq 0$.

Il existe une suite $\left(\alpha_{n}\right)_{n} \in \mathcal{A}$ telle que $\left(\gamma_{n}\right)_{n} \leq\left(\alpha_{n}\right)_{n}$ et telle que $\left(\alpha_{n}\right)_{n}+$ $\left(\alpha_{n}\right)_{n}=\left(\alpha_{n}\right)_{n}$. En effet si l'on pose $P=\Delta\left(\left(\gamma_{n}\right)_{n}\right)$ et pour tout $n>0$, $n P=P+\ldots+P$, alors la partie $Q=\bigcup_{n} n P$ est minorée par 0 et n'est pas majorée. Soit $\varepsilon>0$ et $N=E\left(\varepsilon / \gamma_{1}\right)+1$. Si $n \geq N$ et si $x_{1}+\ldots+x_{n}$ désigne une somme de $n$ éléments de $P$ dont aucun n'est nul, alors $x_{1}+\ldots+x_{n}>\varepsilon$. On en déduit donc que pour tout $n \geq N, n P \cap]-\infty, \varepsilon[=N P \cap]-\infty, \varepsilon[$ et par suite $Q \cap]-\infty, \varepsilon\left[\right.$ est fini (lemme 3). Donc $Q \in \mathcal{O}$ et $\left(\alpha_{n}\right)_{n}=\Delta^{-1}(Q)$ vérifie bien $\left(\gamma_{n}\right)_{n} \leq\left(\alpha_{n}\right)_{n}$ et $\left(\alpha_{n}\right)_{n}+\left(\alpha_{n}\right)_{n}=\left(\alpha_{n}\right)_{n}$. Notons qu'alors $\alpha_{0}=0$.

Inverser $S$ revient à inverser $T$. On choisit un représentant

$$
T=\sum_{n \geq 0} t_{\alpha_{n}} X^{\alpha_{n}}
$$

de $T$ dans $\mathcal{A}_{\left(\alpha_{n}\right)_{n}}$ (on a $t_{\gamma_{0}}=t_{\alpha_{0}} \neq 0$ ) et l'on pose

$$
V=\sum_{n \geq 0} s_{\alpha_{n}} X^{\alpha_{n}}
$$

où la suite $\left(s_{\alpha_{n}}\right)_{n}$ est définie de la manière suivante :

$$
s_{\alpha_{0}}=t_{\alpha_{0}}^{-1} ; \quad \forall n>0, \quad s_{\alpha_{n}}=-t_{\alpha_{0}}^{-1} \sum_{\alpha_{i}+\alpha_{j}=\alpha_{n}, j \neq n} t_{\alpha_{i}} s_{\alpha_{j}} .
$$

On a alors

$$
\left(\sum_{n \geq 0} t_{\alpha_{n}} X^{\alpha_{n}}\right) \cdot\left(\sum_{n \geq 0} s_{\alpha_{n}} X^{\alpha_{n}}\right)=\sum_{n \geq 0}\left(\sum_{\alpha_{i}+\alpha_{j}=\alpha_{n}} t_{\alpha_{i}} s_{\alpha_{j}}\right) X^{\alpha_{n}} .
$$

Cette dernière série vaut 1 par construction et donc $T$ est inversible.

- La loi $\cdot$ est distributive par rapport à + . C'est immédiat.

\subsection{Propriétés}

Lemme 8. L'application $\nu: \coprod \mathcal{A}_{\left(\alpha_{n}\right)_{n}} \rightarrow \mathbb{R} \cup\{\infty\}$ définie par

$$
\nu\left(\sum_{n \geq 0} u_{\gamma_{n}} X^{\gamma_{n}}\right)= \begin{cases}\inf \left\{\gamma_{i}: u_{\gamma_{i}} \neq 0\right\} & \text { si } \exists n_{0} \in \mathbb{N}, u_{\gamma_{n_{0}}} \neq 0, \\ \infty & \text { si } \forall n \in \mathbb{N}, u_{\gamma_{n}} \neq 0,\end{cases}
$$

passe au quotient (i.e. si $S \mathcal{R} S^{\prime}$ alors $\nu(S)=\nu\left(S^{\prime}\right)$ ).

Preuve. Soient $\sum_{n \geq 0} u_{\alpha_{n}} X^{\alpha_{n}}$ et $\sum_{n \geq 0} v_{\beta_{n}} X^{\beta_{n}}$. Le lemme 6 affirme alors que $\left\{r \in \mathbb{Q}: u_{r} \neq 0\right\}=\left\{r \in \mathbb{Q}: v_{r} \neq 0\right\}=E$. On a alors

$$
\nu\left(\sum_{n \geq 0} u_{\alpha_{n}} X^{\alpha_{n}}\right)=\nu\left(\sum_{n \geq 0} v_{\beta_{n}} X^{\beta_{n}}\right)=\min (E) .
$$

Ainsi $\nu$ définit une application de $\widetilde{\text { Puis }}(K) \rightarrow \mathbb{R} \cup\{\infty\}$ que nous continuerons à noter $\nu$. 
ThÉorème 1. Le corps $\widetilde{\mathrm{Puis}}(K)$ est une extension du corps $\operatorname{Puis}(K)$. $L$ 'application $\nu$ est une valuation réelle qui étend la valuation naturelle de $\operatorname{Puis}(K)$.

L'anneau de la valuation $\nu$ est l'anneau des "séries entières de Puiseux généralisées", constitué des séries de Puiseux généralisées admettant un représentant de la forme $\sum_{n \geq 0} u_{\gamma_{n}} X^{\gamma_{n}}$, où $\left(\gamma_{n}\right)_{n}$ vérifie $\gamma_{0} \geq 0$.

Le corps résiduel de $\widehat{\text { Puis }}(K)$ pour $\nu$ est le corps $K$.

Preuve. Considérons le sous-ensemble $\mathcal{B} \subset \mathcal{A}$ constitué des suites $\left(\alpha_{n}\right)_{n}$ de la forme

$$
\alpha_{n}=\frac{n-a}{n_{0}}, \quad a \in \mathbb{Z}, n \in \mathbb{N}^{*} .
$$

Le sous-système d'ensembles et d'applications $\left\{\mathcal{A}_{\left(\alpha_{n}\right)_{n}}, \Phi_{\left(\alpha_{n}\right)_{n},\left(\beta_{n}\right)_{n}}\right\}_{\mathcal{B}}$ est le même que celui qui nous a permis de définir, dans la partie 1 , le corps Puis $(K)$. D'où, par passage au quotient, l'extension $\widetilde{\operatorname{Puis}}(K) / \operatorname{Puis}(K)$.

Il est clair que pour tout $S \in \widetilde{\operatorname{Puis}}(K), \nu(S)=\infty \Leftrightarrow S=0$. Soient maintenant $S$ et $T$ dans $\widetilde{\text { Puis }}(K)$; si l'on choisit pour représentant de $S$ et $T$ des séries $\sum_{n \geq 0} u_{\alpha_{n}} X^{\alpha_{n}}$ et $\sum_{n \geq 0} v_{\beta_{n}} X^{\beta_{n}}$ telles que $u_{\alpha_{0}} \neq 0$ et $u_{\beta_{0}} \neq 0$, alors $\nu(S T)=\alpha_{0}+\beta_{0}=\nu(S)+\nu(T)$.

Si l'on choisit maintenant pour représentant de $S$ et $T$ des séries $\sum_{n \geq 0} u_{\alpha_{n}} X^{\alpha_{n}}$ et $\sum_{n \geq 0} v_{\alpha_{n}} X^{\alpha_{n}}$, on a

$$
\begin{aligned}
\nu(S+T) & =\min \left(\alpha_{n}: u_{\alpha_{n}}+v_{\alpha_{n}} \neq 0\right) \\
& \geq \inf \left(\min \left(\alpha_{n}: u_{\alpha_{n}} \neq 0\right) ; \min \left(\alpha_{n}: v_{\alpha_{n}} \neq 0\right)\right) \\
& =\inf (\nu(S), \nu(T)),
\end{aligned}
$$

ce qui assure que $\nu$ est une valuation réelle sur $\widetilde{\text { Puis }}(K)$. Il est clair que la restriction de $\nu$ à $\operatorname{Puis}(K)$ est bien la valuation usuelle.

Notons $A_{\nu}$ l'anneau de la valuation $\nu$ et $M_{\nu}$ l'idéal de la valuation. Les éléments de $A_{\nu}$ (resp. $M_{\nu}$ ) admettent tous des représentants de la forme $\sum_{n \geq 0} u_{\alpha_{n}} X^{\alpha_{n}}$ tels que $\alpha_{0}=0$ (resp. $\alpha_{0}=0$ et $u_{\alpha_{0}}=0$ ). Remarquons qu'alors $K$ se plonge dans $A_{\nu} / M_{\nu}$ et que si $S=\sum_{n \geq 0} u_{\alpha_{n}} X^{\alpha_{n}} \in A_{\nu}\left(\alpha_{0}=\right.$ $0)$, alors $S-x \in M_{\nu}$ avec $x=u_{\alpha_{0}} \in K$ et donc que $A_{\nu} / M_{\nu}=K$.

ThÉORÈme 2. Le corps valué ( $\widetilde{\text { Puis }}(K), \nu)$ est complet; c'est le complété de $\operatorname{Puis}(K)$ pour la valuation usuelle.

Preuve. $\widetilde{\text { Puis }}(K)$ est complet pour $\nu$. En effet, soit $\left(S_{l}\right)_{l}$ une suite de Cauchy dans $\widetilde{\text { Puis }}(K)$. Pour chaque $l \in \mathbb{N}$, on associe à $S_{l}$ son représentant canonique $S_{l}=\sum_{n \geq 0} u_{l_{n}} X^{l_{n}}$. Soit $H \in \mathbb{Z}$; il existe $h \in \mathbb{N}$ tel que pour tout $l \geq h, \nu\left(S_{l}-\bar{S}_{h}\right) \geq H$. Soit alors $n_{H}=\sup \left\{n \in \mathbb{N}: l_{n} \leq H\right.$ et $\left.h_{n} \leq H\right\}$; on a, pour tout $n=0, \ldots, n_{H}, l_{n}=h_{n}$ et $u_{l_{n}}=u_{h_{n}}$. Comme $\lim _{H \rightarrow+\infty} n_{H}=+\infty$, on obtient alors une suite $\left(\alpha_{n}\right)_{n} \in \mathcal{A}$ et une suite 
$\left(u_{\alpha_{n}}\right)_{n}$ d'éléments de $K$ telles que si $S=\sum_{n \geq 0} u_{\alpha_{n}} X^{\alpha_{n}}$, alors pour tout $l \geq h, \nu\left(S-S_{l}\right) \geq H$. Ainsi $\left(S_{l}\right)_{l}$ converge vers $S$.

Pour toute série $S=\sum_{n \geq 0} u_{\alpha_{n}} X^{\alpha_{n}}$, il existe une suite de séries de Puiseux $\left(S_{n}\right)_{n}$ qui converge vers $S$. En effet, il suffit de considérer pour $n$ donné la série (finie) $S_{n}=\sum_{k=0}^{n} u_{\alpha_{k}} X^{\alpha_{k}}$. Il est clair que (avec les notation de la partie 1)

$$
S_{n} \in K_{\text {p.p.c.m. des dénominateurs des } \alpha_{k} \subset \operatorname{Puis}(K) .}
$$

La suite $\left(S_{n}\right)_{n}$ converge bien vers $S$, car $\nu\left(S-S_{n}\right) \geq \alpha_{n+1} \rightarrow+\infty$.

Rappelons (voir [Ami]) que si $(C, \nu)$ est un corps valué algébriquement clos, alors son complété $(\widetilde{C}, \nu)$ est algébriquement clos. Par ailleurs, si $(K, \nu)$ est un corps local alors il existe une unique valuation sur $\bar{K}$ qui prolonge $\nu$. On en déduit donc que si $(K, \nu)$ est un corps valué et que $\nu$ est discrète, alors le plus petit corps complet et algébriquement clos (ce que nous appellerons "la fermeture" de $K)$ est le corps $(\stackrel{\widetilde{\widetilde{K}}}{,} \nu)$ où $\nu$ est une valuation définie sans équivoque d'après ce qui précède.

Appliquons ces quelques remarques au corps $\bar{K}(X)$ où $\bar{K}$ désigne un corps algébriquement clos de caractéristique 0. On a alors $\widetilde{K(X)}=\bar{K}((X))$ et (cf. [Ser]), $\overline{\bar{K}((X))}=$ Puis $(\bar{K})$. D'après l'étude menée précédemment, on en déduit que la fermeture de $\bar{K}(X)$ est le corps $\widetilde{\operatorname{Puis}}(\bar{K})$.

Remarque. Cette construction est analogue à celle des corps $\mathbb{C}_{p}$ (l'analogie identifiant le corps $K(X)$ au corps $\mathbb{Q}$ ). Bien évidemment, cette analogie, en termes arithmétiques, est moins saisissante que celle entre $\mathbb{F}_{p}(T)$ et $\mathbb{Q}$.

Quand $K$ n'est pas algébriquement clos (mais reste de caractéristique 0 ), on ne peut pas conclure immédiatement car, dans ce cas, $\overline{K((X))}$ n'est pas un corps de séries de Puiseux (voir [Des] et le théorème suivant). On a pourtant :

ThÉORÈme 3. Si $K$ désigne un corps de caractéristique nulle, la fermeture de $K((X))$ est égale à $\widetilde{\text { Puis }}(\bar{K})$.

Preuve. On commence par établir le résultat suivant :

Lemme 9. Soit $K$ un corps de caractéristique 0. La clôture algébrique de $K((X))$ est le corps $\bigcup_{L / K \text { finie }} \operatorname{Puis}(L)$.

Pre u ve. Le corps Puis $(\bar{K})$ est algébriquement clos et contient $K((X))$. Soit alors $S=\sum_{k \geq k_{0}} a_{k} X^{k / n_{0}}$ une série de Puiseux algébrique sur $K((X))$. Supposons que le corps $L=K\left(a_{k}\right)_{k}$ soit de dimension infinie sur $K$. Soit $I$ l'ensemble des $K$-isomorphismes de $L$. Comme $K$ est de caractéristique nulle, $L / K$ est séparable et par suite $\sharp I=+\infty$. Soit $\sigma \in I ; \sigma$ se relève en un $K((X))$-automorphisme de Puis $(\bar{K})$ par action sur les coefficients des séries 
de Puiseux. Mais alors, si $\sigma, \tau$ sont deux éléments distincts de $I$ remontés à Puis $(\bar{K})$, on a $\sigma(S) \neq \tau(S)$ car la famille $\left(a_{k}\right)_{k}$ engendre $L$ sur $K$. Ainsi $S$ possède une infinité de conjugués, ce qui est absurde.

Réciproquement, si $L / K$ est finie, alors $\operatorname{Puis}(L) / \operatorname{Puis}(K)$ est finie; on a d'ailleurs $[\operatorname{Puis}(L): \operatorname{Puis}(K)]=[L: K]$. Comme Puis $(K) / K((X))$ est une extension algébrique, on en déduit que $\mathrm{Puis}(L) / K((X))$ en est une aussi.

Revenons à la preuve du théorème 3 . On sait que $\widetilde{\text { Puis }}(\bar{K})$ est un corps complet et algébriquement clos. Pour prouver le théorème, il suffit alors de montrer que Puis $(\bar{K})$ est contenu dans le complété du corps $\bigcup_{L / K \text { finie }} \operatorname{Puis}(L)$.

Soit $S=\sum_{n>0} u_{\alpha_{n}} X^{\alpha_{n}} \in \widetilde{\text { Puis }}(\bar{K})$; la suite $S_{n}=\sum_{k=0}^{n} u_{\alpha_{k}} X^{\alpha_{k}}$ vérifie bien $\lim _{n} S_{n}=S$ et, par ailleurs, $S_{n} \in \operatorname{Puis}(L)$ avec

$$
L=K\left(u_{\alpha_{0}}, \ldots, u_{\alpha_{n}}\right)
$$

qui est un corps de dimension fini sur $K$. Ceci achève la preuve.

En paraphrasant N. Koblitz (cf. [Kob]) qui parlait des corps $\mathbb{C}_{p}$, nous concluerons en disant que $\widetilde{\text { Puis }}(\bar{K})$ est un merveilleux royaume où vit l'analyse de $K((X))$.

\section{Références}

[Ami] Y. Amice, Les nombres p-adiques, Presses Univ. France, 1975.

[Des] B. Deschamps, Clôture totalement réelle des corps de nombres ordonnables, Manuscripta Math. 100 (1999), 277-289.

[Kob] N. Koblitz, p-adic Numbers, p-adic Analysis and Zeta-Functions, Springer, 1996.

[Ser] J. P. Serre, Corps Locaux, Hermann, 1968.

Equipe de théorie des nombres

Faculté des sciences et techniques

Université Jean Monnet

23 rue du docteur Paul Michelon

42023 Saint-Etienne, Cedex 2, France

E-mail: Bruno.Deschamps@univ-st-etienne.fr 